\title{
Efficacy of manual dissection of lymph nodes in colon cancer resections
}

\author{
Henry G Brown ${ }^{1}$, Todd M Luckasevic ${ }^{1}$, David S Medich², James P Celebrezze ${ }^{2}$ and \\ Sandra M Jones ${ }^{2}$ \\ ${ }^{1}$ Department of Pathology and ${ }^{2}$ Department of Surgery, Allegheny General Hospital, Pittsburgh, PA, USA
}

\begin{abstract}
The adequacy of lymph node dissection of colonic resection specimens influences the clinical and pathologic staging, leading to important postsurgical treatment decisions. Although manual lymph node dissection is the current standard at most institutions, recent statistical studies indicate that all lymph nodes, including those measuring 1-2 $\mathrm{mm}$, should be recovered to be assured of lymph node negative status. Thus, we tested the efficacy of gross dissection by submitting the entire residual mesenteric fat. We analyzed 15 randomly chosen colonic resections (2 pT1, 1 pT2, 11 pT3, 1 pT4). After standard gross dissection of lymph nodes and submission of colonic material for diagnosis, the entire remaining mesenteric material was dehydrated over several days by serial washing in alcohol and acetone. All of the mesenteric tissue was submitted for histology. The average number of nodes found by original gross inspection was 20.8, while the average number of additional nodes found after clearing was 68.6 . In all, $83 \%$ of the additional nodes were $2.0 \mathrm{~mm}$ or less in size. There were seven pNO cases; one was upstaged by additional findings that may have been artifactual. There were four pN1 cases; three were upstaged to pN2 after submission of the mesenteric material. All four pN2 tumors had additional metastases identified. In all, $75 \%$ of all positive nodes were under $2.0 \mathrm{~mm}$ in size. In this limited sample, standard gross dissection proved sufficient for most pNO tumors to remain node negative. However, our findings within the pN1 group show that examination of all of the mesenteric material may be necessary to be assured of correct $\mathrm{pN}$ status.
\end{abstract}

Modern Pathology (2004) 17, 402-406, advance online publication, 13 February 2004; doi:10.1038/modpathol.3800071

Keywords: colon; adenocarcinoma; lymph node; clearing; dissection; staging; prognosis

The presence or absence of lymph node metastasis is pivotal for predicting the clinical outcome of patients who have undergone radical surgery for colorectal carcinoma. ${ }^{1}$ Lymph nodes are an integral component of the TNM classification system, a major determinant of adjunct therapy, and a prognostic marker in colorectal adenocarcinoma. ${ }^{2}$ Recovering a greater number of lymph nodes guards against missing a lymph node metastasis and allows for more accurate staging of patients. The identification of a single lymph node metastasis is sufficient to offer adjunctive therapy. The most important determinant of survival is the presence or absence of metastases in regional lymph nodes. ${ }^{3}$ Thus, the detection and examination of the largest possible number of lymph nodes are essential for correct staging, therapeutic decisions, and prognosis.,

Correspondence: HG Brown, MD, PhD, Department of Pathology, Loyola University Medical Center, 2160 South First Avenue, Maywood, IL 60153, USA. E-mail: hbrown@lumc.edu

Received 25 August 2003; revised 30 October 2003; accepted 5 December 2003; published online 13 February 2004
Despite claims of a meticulous search for lymph nodes in surgical specimens, wide variations in the number of total nodes and lymph node metastases continue to exist. ${ }^{2,6,7}$ This variation may be due to the size of the specimen, the number of regional lymph nodes present in the specimen, and the number of nodes with metastases present. ${ }^{3,8}$ Pathologists also vary in their diligence, skill, and patience in dissecting the lymph nodes of a surgical specimen. ${ }^{9}$

In this study, we enrolled 15 randomly chosen colon resections, which were predominantly a tumor stage of T3. After manual gross lymph node dissection, the mesenteric fat was dehydrated and all the remaining tissue was submitted for microscopic examination. We compared the total number of nodes found during a standard gross dissection with the number found after submission of the entire mesentery. The additional number of metastases and potential effect on the cancer stage was also investigated. We measured the size of the additional nodes found, to determine if the additional nodes were large enough such that they 
should have been found with a reasonably diligent dissection.

\section{Materials and methods}

We analyzed 15 randomly chosen colonic adenocarcinoma resections (Table 1). After standard gross dissection of lymph nodes and submission of colonic material for diagnosis, the entire mesenteric material was dehydrated in situ over several days of washing in a graduated series of alcohol and acetone. Between each daily washing, the fat was placed between two large surgical pads and lightly pressed with a rolling pin to express fully all of the previous solution. After $100 \%$ acetone washing, the entire remaining specimen was placed into cassettes and allowed to soak in xylene for an additional $24 \mathrm{~h}$ prior to histologic processing. We used this method of slow dehydration for two reasons. First, processing the fatty tissue prior to cassette placement reduced the volume of material by approximately two-thirds. Second, this method ensured that the tissue would be well dehydrated and thus easier to embed and cut. We noted no significant difference between the quality of our sections and that of normally processed fatty tissue.

The surgical blocks were cut and stained with hematoxylin and eosin. A single microscopic section was examined independently by two pathologists (TML and HGB). The number of lymph nodes, size of the nodes, and any metastases were recorded for each case. This protocol was approved by our institution's Institutional Review Board (IRB).

\section{Results}

The average length of the resection was 28 . $0 \pm 9.0 \mathrm{~cm}$, and the average number of lymph nodes found with gross inspection was $20.9 \pm 12.5$ with a range of 5-47 nodes. There was no correlation between the length of resection and the number of nodes found after manual dissection $(r=0.04)$. The average number of additional nodes found after complete submission was $68.6 \pm 28.0$, with a range of 37-112. There was no correlation between the number of nodes found with gross dissection and the number found after complete submission $(r=0.18)$. However, there was a moderate correlation between the length of the resection and the number of nodes found after complete submission $(r=0.43)$. In all, $82 \%$ of the additional nodes were below $2.0 \mathrm{~mm}$ in size (Figure 1).

There were seven pNo cases, one of which (a pT4) was upstaged by additional findings that may have been artifactual. In this last case, we did not identify any lymph nodes with metastases. This case was staged as a pT4 because of local abdominal wall invasion. The soft-tissue metastases identified were intimately involved with skeletal tissue, and we believe that these areas represented overvigorous dissection by the prosector rather than true metastatic implants. There were four pN1 cases, three of

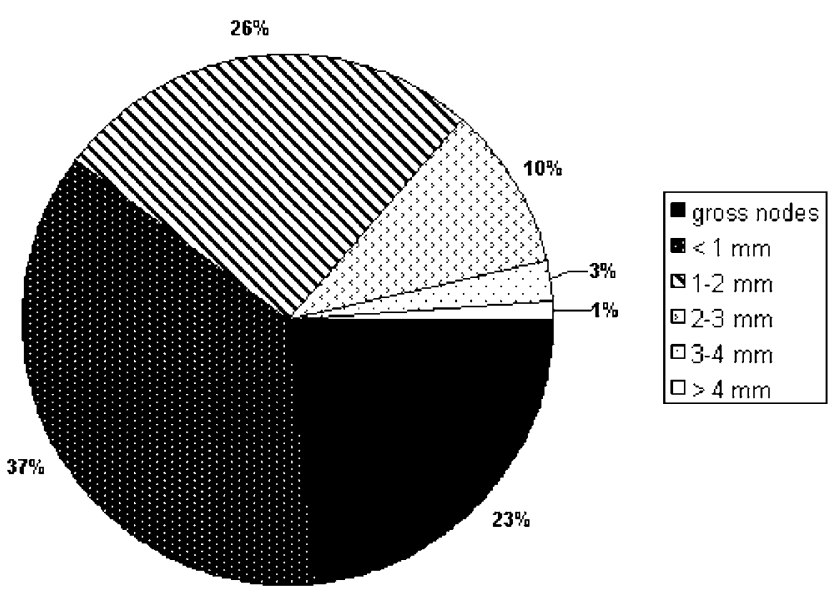

Figure 1 Average percent contribution of lymph nodes per size. Note that if gross nodes (23\% of the total) are not considered, $82 \%$ of the remaining nodes are smaller than $2 \mathrm{~mm}$.

Table 1 Characteristics of resection specimens

\begin{tabular}{|c|c|c|c|c|c|c|}
\hline Case & Age (years) & Sex & Location & T Grade & Tumor size (cm) & Resection length $(\mathrm{cm})$ \\
\hline 1 & 72 & $\mathrm{~m}$ & Right & T3 & 3.0 & 17.0 \\
\hline 2 & 79 & $\mathrm{f}$ & Left & T3 & 7.6 & 31.0 \\
\hline 3 & 48 & $\mathrm{f}$ & Sigmoid & $\mathrm{T} 1$ & 2.0 & 14.0 \\
\hline 4 & 72 & $\mathrm{~m}$ & Sigmoid & $\mathrm{T} 1$ & 1.2 & 48.0 \\
\hline 5 & 80 & $\mathrm{~m}$ & Right & $\mathrm{T} 2$ & 3.0 & 25.0 \\
\hline 6 & 63 & $\mathrm{~m}$ & Right & Т3 & 8.0 & 38.0 \\
\hline 7 & 63 & $\mathrm{~m}$ & Left & $\mathrm{T} 4$ & 4.5 & 28.0 \\
\hline 8 & 64 & $\mathrm{~m}$ & Sigmoid & T3 & 3.0 & 30.0 \\
\hline 9 & 79 & $\mathrm{f}$ & Right & T3 & 2.6 & 27.0 \\
\hline 10 & 71 & $\mathrm{~m}$ & Sigmoid & T3 & 3.9 & 34.0 \\
\hline 11 & 63 & $\mathrm{~m}$ & Right & T3 & 5.0 & 26.0 \\
\hline 12 & 64 & $\mathrm{f}$ & Sigmoid (two lesions) & T3 & $3.0,1.6$ & 37.0 \\
\hline 13 & 72 & $\mathrm{~m}$ & Right & T3 & 6.5 & 28.0 \\
\hline 14 & 74 & $\mathrm{f}$ & Right & T3 & 3.2 & 14.0 \\
\hline 15 & 49 & $\mathrm{f}$ & Sigmoid & T3 & 1.5 & 26.0 \\
\hline
\end{tabular}


which were upstaged to $\mathrm{pN} 2$ after submission of the mesenteric material. All four pN2 tumors had additional metastases in the extra material. In all, $75 \%$ of all positive nodes were under $2.0 \mathrm{~mm}$ (Tables 2 and 3 , Figure 2).

\section{Discussion}

There has been much debate in the literature about the minimum number of lymph nodes necessary to stage colorectal tumors adequately. There is no consensus on the minimum number of lymph nodes needed for proper staging, although the current recommendation is to harvest at least $12 .{ }^{10} \mathrm{Cur}$ rently, the pathologist usually obtains lymph nodes for histologic study after their recognition by sight and palpation. This method for recovering lymph nodes is termed the manual method.

A second method, designated as a 'lymph node clearing technique,' treats the surgical specimen by dissolving the fat. The result is a translucent mesentery that preserves lymph node structure. Nodes as small as $1.0 \mathrm{~mm}$ may be dissected out of the cleared fat. The usefulness of this method for detecting lymph node metastases of carcinoma of the colon and rectum was reported as early as 1938. ${ }^{11}$ Clearing significantly increases the number of harvested lymph nodes in the specimens, and thus the accuracy of tumor staging. ${ }^{1}$ The disadvantages of this technique are the turn-around time, cost, and labor intensity, ${ }^{1,12}$ as well as exposure to xylene.

In this study, we used a variation of the clearing technique, which we refer to as 'complete submission of the mesentery.' Instead of dehydration and then clearing in xylene, the mesentery is dehydrated over a period of 1 week. Unlike lymph node clearing, where lymph nodes are dissected from the residual soft tissue before submission, no additional gross examination of the dehydrated specimen was carried out prior to tissue submission. Instead, all of the mesenteric material is submitted for histology. This technique decreased the time and effort of the prosector, and also guaranteed that even very small lymph nodes less than $1.0 \mathrm{~mm}$ in size would be found. A drawback of this method, however, is the additional time and effort invested by the histotechnologist.

There was a lack of correlation between the number of gross nodes found and the length of the resection, as well as a lack of correlation between the numbers of lymph nodes found in manual dissection vs total submission. This is in contrast to the moderate correlation between the number of nodes found after clearing and resection length. We believe this implies that total submission does not simply show more nodes, but gives a more accurate

Table 3 Size characteristics of additional nodes (number of positive nodes)

\begin{tabular}{rcrrll}
\hline Case & $</=1 \mathrm{~mm}$ & $1-2 \mathrm{~mm}$ & $2-3 \mathrm{~mm}$ & $3-4 \mathrm{~mm}$ & $4+\mathrm{mm}$ \\
\hline 1 & 24 & 12 & 5 & 2 & 2 \\
2 & 39 & 38 & 8 & 2 & 1 \\
3 & 14 & 15 & 16 & 8 & 2 \\
4 & 13 & 34 & 15 & 6 & 0 \\
5 & 34 & 35 & 10 & 2 & 0 \\
6 & 52 & 39 & 11 & 0 & 3 \\
7 & 60 & 18 & 6 & 0 & 0 \\
8 & 44 & 22 & 13 & 3 & 1 \\
9 & 24 & $20(2)$ & 6 & 2 & 0 \\
10 & $82(1)$ & $26(2)$ & 3 & 1 & 0 \\
11 & 50 & $35(3)$ & 12 & 5 & 3 \\
12 & $14(1)$ & $9(3)$ & $9(4)$ & $2(1)$ & $3(3)$ \\
13 & 23 & $20(2)$ & $8(2)$ & 0 & 0 \\
14 & 3 & 9 & 3 & 2 & 0 \\
15 & 20 & 20 & 6 & 0 & 0 \\
\hline
\end{tabular}

Table 2 Comparison of node number and stage between manual dissection and complete submission

\begin{tabular}{|c|c|c|c|c|c|}
\hline Case & $\begin{array}{l}\text { Number of nodes } \\
\text { found by manual } \\
\text { dissection }\end{array}$ & $\begin{array}{l}\text { Manual pN } \\
\text { (number of positive } \\
\text { nodes) }\end{array}$ & $\begin{array}{c}\text { Number of additional } \\
\text { nodes found after total } \\
\text { submission }\end{array}$ & Did $p N$ change? & $\begin{array}{l}\text { Final pN (additional } \\
\text { number of positive } \\
\text { nodes found) }\end{array}$ \\
\hline 1 & 17 & No & 45 & No & No \\
\hline 2 & 18 & No & 88 & No & No \\
\hline 3 & 9 & No & 55 & No & No \\
\hline 4 & 10 & No & 68 & No & No \\
\hline 5 & 47 & No & 81 & No & No \\
\hline 6 & 14 & No & 105 & No & No \\
\hline 7 & 12 & No & 84 & YES & N1 (3 possible STM) \\
\hline 8 & 5 & N1 (1) & 83 & No & N1 \\
\hline 9 & 23 & N1 (3) & 52 & YES & N2 (2) \\
\hline 10 & 23 & N1 (2) & 112 & YES & N2 (3 and 3 STM) \\
\hline 11 & 20 & N1 (1) & 105 & YES & N2 (3) \\
\hline 12 & 46 & N2 (11) & 37 & No & N2 (12) \\
\hline 13 & 18 & N2 (6) & 51 & No & N2 (4) \\
\hline 14 & 17 & N2 (5) & 17 & No & N2 (2 STM) \\
\hline 15 & 34 & N2 (17) & 46 & No & N2 (multiple STM) \\
\hline
\end{tabular}

STM $=$ Soft-tissue metastases. 


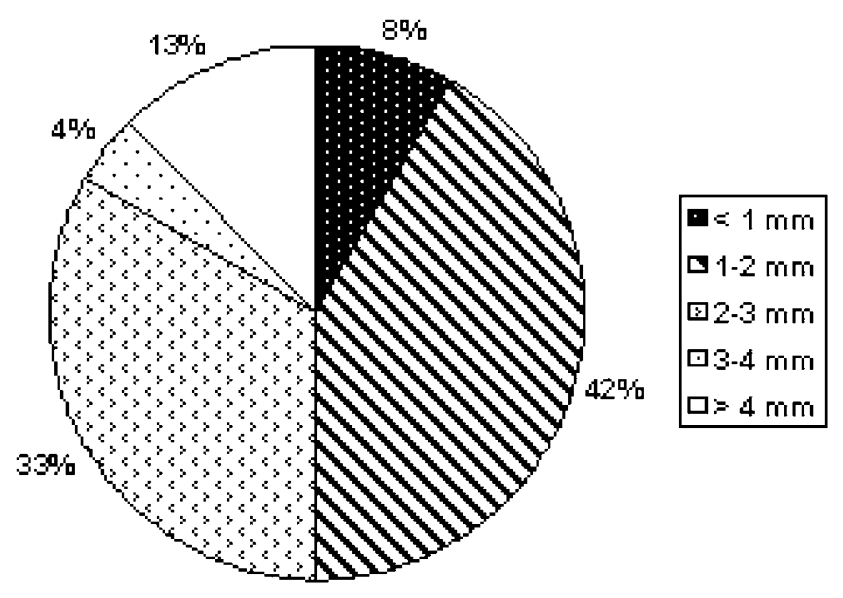

Figure 2 Percentage of additional positive lymph nodes (excluding soft-tissue metastases) by size. This pie chart represents the five cases of a total of 15 that had additional lymph node metastases. Three cases that had soft-tissue metastases only are not included in this analysis. In all, $83 \%$ of the additional positive lymph nodes are below $3 \mathrm{~mm}$ in size, and in fact, all of the larger positive lymph nodes came from the same case that was already known to be a pN2 (Table 3, case 12).

assessment of the mesenteric lymphatic system as it correlates with specimen size and not the number of nodes found by manual inspection.

Almost $50 \%$ of the additional nodes found in our study were below $1 \mathrm{~mm}$, and $82 \%$ of them were below $2 \mathrm{~mm}$ (Figure 1). Many of these nodes may have been imperceptible even with clearing techniques, hence the importance of submission of all of the mesenteric adipose tissue.

It is significant that the additional positive lymph nodes metastases identified in our study do not represent 'micrometastases' which would be apparent only with adjunctive techniques. We have no doubt that using immunoperoxidase, PCR, or other methods would have revealed more positive metastases, but would have added expense and increased the time delay in diagnosis. ${ }^{13-15}$

Standard gross dissection was sufficient for most pNo tumors to remain node negative (Table 2). This held true even in the cases with 'insufficient' gross lymph node dissections of under 12 lymph nodes recovered. We upgraded only one pNo case, a pT4 (Table 2, case 7). In this case, no lymph node metastases were found, and we believe that the softtissue implants seen may have been an artifact of dissection around the abdominal wall invasion. The complete submission technique may not be appropriate in T4 tumors where the prosector cannot reliably dissect the mesenteric tissue away from the locally invasive tumor. Three of four pN1 tumors were upstaged to pN2 with this protocol, and none of the additional positive nodes was larger than $3.0 \mathrm{~mm}$ (Tables 2 and 3, cases 9,10, and 11). All three of the cases had more than 20 lymph nodes submitted with gross dissection; thus manual dissection would have been regarded as adequate.
Finally, every pN2 case had either additional positive lymph nodes or soft-tissue metastases (Table 2).

It is worthwhile to compare our findings to the previous work by Scott and Grace ${ }^{16}$ and Scott et al. ${ }^{17}$ In these studies, a total of 103 separate specimens were examined in a manner similar to the present study, with a primary gross dissection and then a secondary submission of nodes after using clearing techniques. After clearing, five of their 103 specimens were found to be lymph node positive. Of these five cases, all would have been reclassified as pN1. Follow-up 5 years later revealed that four of the five patients with upgraded specimens had died of disease. Scott's study and others indicate that up to $8 \%$ of pNo cases could be upgraded after lymph node clearing, ${ }^{9,16,18}$ and it is somewhat unexpected that we did not identify any positive lymph nodes in our pNo cases; this may have been due to our small sample size.

Over the last decade, the appropriate number of nodes to sample in colonic resections has ranged from six to 17, with the current consensus being 12$15 .^{1,3,7,10,12,1719-23}$ Our study supports the contention of Tepper et $a l^{12}$ that more uniform standards in lymph node assessment are necessary to prevent systematic undergrading of lymph node status, which may be a determining factor in treatment failure of pT3 pNo and pN1 tumors.

Our sample size is not large enough for us to come to a definitive conclusion about the minimum number of nodes necessary to validate a pNo diagnosis, but in the case of an inadequate gross dissection, clearing, or as described in this paper, total submission of mesenteric fat, remain important viable alternatives to a second gross dissection, and, indeed, may become necessary if 30 or more lymph nodes are expected to be analyzed routinely, as has been suggested by Goldstein ${ }^{2}$ and Joseph et al. ${ }^{24} \mathrm{We}$ believe that our study reveals that the complete submission technique is more accurate in sampling the mesenteric lymphatic system than standard manual dissection. However, clinical validation must be performed before routinely advocating this process.

\section{References}

1 Cianchi F, Palomba A, Boddi V, et al. Lymph node recovery from colorectal tumor specimens: recommendation for a minimum number of lymph nodes to be examined. World J Surg 2002;26:384-389.

2 Goldstein NS. Lymph node recoveries from 2427 pT3 colorectal resection specimens spanning 45 years: recommendations for a minimum number of recovered lymph nodes based on predictive probabilities. Am J Surg Pathol 2002;26:179-189.

3 Swanson RS, Compton CC, Stewart AK, et al. The prognosis of T3No colon cancer is dependent on the number of lymph nodes examined. Ann Surg Oncol 2003;10:65-71. 
4 Compton CC, Fielding LP, Burgart LJ, et al. Prognostic factors in colorectal cancer. College of American Pathologists Consensus Statement 1999. Arch Pathol Lab Med 2000;124:979-994.

5 Koren R, Siegal A, Klein B, et al. Lymph noderevealing solution: simple new method for detecting minute lymph nodes in colon carcinoma. Dis Colon Rectum 1997;40:407-410.

6 Herrera-Ornelas L, Justiniano J, Castillo N, et al. Metastases in small lymph nodes from colon cancer. Arch Surg 1987;122:1253-1256.

7 Maurel J, Launoy G, Grosclaude P, et al. Lymph node harvest reporting in patients with carcinoma of the large bowel: a French population-based study. Cancer 1998;82:1482-1486.

8 Haboubi NY, Abdalla SA, Amini S, et al. The novel combination of fat clearance and immunohistochemistry improves prediction of the outcome of patients with colorectal carcinomas: a preliminary study. Int J Colorectal Dis 1998;13:99-102.

9 Haboubi NY, Clark P, Kaftan SM, et al. The importance of combining xylene clearance and immunohistochemistry in the accurate staging of colorectal carcinoma. J R Soc Med 1992;85:386-388.

10 Compton CC. Updated protocol for the examination of specimens from patients with carcinomas of the colon and rectum, excluding carcinoid tumors, lymphomas, sarcomas, and tumors of the vermiform appendix: a basis for checklists. Cancer Committee. Arch Pathol Lab Med 2000;124:1016-1025.

11 Gilchrist R, David V. Lymphatic spread of carcinoma of the rectum. Ann Surg 1938;108:621-642.

12 Tepper JE, O’Connell MJ, Niedzwiecki D, et al. Impact of number of nodes retrieved on outcome in patients with rectal cancer. J Clin Oncol 2001;19:157-163.

13 Liefers GJ, Cleton-Jansen AM, van de Velde CJ, et al. Micrometastases and survival in stage II colorectal cancer. N Engl J Med 1998;339:223-228.

14 Merrie AE, van Rij AM, Dennett ER, et al. Prognostic significance of occult metastases in colon cancer. Dis Colon Rectum 2003;46:221-231.
15 Belly RT, Rosenblatt JD, Steinmann M, et al. Detection of mutated K12-ras in histologically negative lymph nodes as an indicator of poor prognosis in stage II colorectal cancer. Clin Colorectal Cancer 2001;1: 110-116.

16 Scott KW, Grace RH. Detection of lymph node metastases in colorectal carcinoma before and after fat clearance. Br J Surg 1989;76:1165-1167.

17 Scott KW, Grace RH, Gibbons P. Five-year follow-up study of the fat clearance technique in colorectal carcinoma. Dis Colon Rectum 1994;37:126-128.

18 Cohen SM, Wexner SD, Schmitt SL, et al. Effect of xylene clearance of mesenteric fat on harvest of lymph nodes after colonic resection. Eur J Surg 1994;160: 693-697.

19 Fielding LP, Arsenault PA, Chapuis PH, et al. Clinicopathological staging for colorectal cancer: an International Documentation System (IDS) and an International Comprehensive Anatomical Terminology (ICAT). J Gastroenterol Hepatol 1991;6:325-344.

20 Goldstein NS, Sanford W, Coffey M, et al. Lymph node recovery from colorectal resection specimens removed for adenocarcinoma. Trends over time and a recommendation for a minimum number of lymph nodes to be recovered. Am J Clin Pathol 1996;106: 209-216.

21 Hernanz F, Revuelta S, Redondo C, et al. Colorectal adenocarcinoma: quality of the assessment of lymph node metastases. Dis Colon Rectum 1994;37: 373-376.

22 Wong JH, Severino R, Honnebier MB, et al. Number of nodes examined and staging accuracy in colorectal carcinoma. J Clin Oncol 1999;17:2896-2900.

23 Wong JH, Bowles BJ, Bueno R, et al. Impact of the number of negative nodes on disease-free survival in colorectal cancer patients. Dis Colon Rectum 2002;45:1341-1348.

24 Joseph NE, Sigurdson ER, Hanlon AL, et al. Accuracy of determining nodal negativity in colorectal cancer on the basis of the number of nodes retrieved on resection. Ann Surg Oncol 2003;10:213-218. 